\title{
Exosomes in stroke pathogenesis and therapy
}

\author{
Zheng Gang Zhang' and Michael Chopp ${ }^{1,2}$ \\ 'Department of Neurology, Henry Ford Hospital, Detroit, Michigan, USA. ²Department of Physics, Oakland University, Rochester, Michigan, USA
}

\begin{abstract}
Stroke is one of the leading causes of death and disability worldwide. Stroke recovery is orchestrated by a set of highly interactive processes that involve the neurovascular unit and neural stem cells. Emerging data suggest that exosomes play an important role in intercellular communication by transferring exosomal protein and RNA cargo between source and target cells in the brain. Here, we review these advances and their impact on promoting coupled brain remodeling processes after stroke. The use of exosomes for therapeutic applications in stroke is also highlighted.
\end{abstract}

\section{Introduction}

Stroke is one of leading causes of death and disability worldwide (1). Since it was approved by the FDA in 1996, tissue plasminogen activator (tPA) has become the only treatment for patients presenting with stroke within 4.5 hours of onset (2-5). Recently, endovascular thrombectomy has been shown to extend the therapeutic window for patients to within 12 hours after stroke onset by rapidly recanalyzing the occluded blood vessels and reestablishing tissue perfusion, indicating that early tissue reperfusion can salvage dying brain cells (6-9). However, restoration of cerebral blood flow (CBF) beyond a critical time point cannot rescue irreversibly damaged brain cells, which leads to long-term disability in a large population of stroke survivors (9). High risk of brain hemorrhage also limits the tPA treatment to a small percentage of patients with ischemic stroke. Thus, there is a compelling need to develop therapies for early brain tissue perfusion after acute stroke and for enhancement of functional recovery in stroke survivors.

The neurovascular unit includes endothelial cells, vascular smooth muscle, glia, neurons, and associated tissue matrix proteins (9). Stroke induces highly dynamic alterations of the neurovascular unit, which contribute to the development of brain injury (9). During stroke recovery, ischemic brain undergoes limited remodeling, with a set of highly interactive processes involving components of the neurovascular unit and neural stem cells $(10,11)$.

Exosomes are endosome-derived small membrane vesicles, approximately 30 to $100 \mathrm{~nm}$ in diameter, and are released into extracellular fluids by cells in all living systems $(12,13)$. They are present in biofluids such as blood and the cerebrospinal fluid (CSF) (13). Exosomes carry proteins, lipids, and genetic materials and play essential roles in intercellular communication by transferring their cargo between source and target cells under physiological and pathophysiological conditions $(12,13)$. Emerging data indicate that exosomes regulate intercellular communication among components of the neurovascular unit after stroke. In this Review, we highlight recent insights into the role of exosomes and exosomal microRNAs (miRNAs) in brain repair processes after stroke and discuss potential applications of exosomes for stroke therapy.

Conflict of interest: The authors have declared that no conflict of interest exists. Reference information: J Clin Invest. 2016;126(4):1190-1197. doi:10.1172/JCI81133.

\section{Pathophysiology of ischemic stroke}

Ischemic stroke is primarily caused by thromboembolic occlusion of a major artery that supplies the brain (9). When emboli or thrombi in situ occlude cerebral arteries, circulating platelets are rapidly recruited to the site of the occluded vessels (14). Platelets, along with thrombin and fibrin resulting from tissue factoractivated blood coagulation, are the primary contributors to thrombus development at the site of the occluded artery $(14,15)$. Concomitantly, occlusion of a major cerebral artery triggers development of secondary thrombosis in downstream microvessels, which causes dysfunction of cerebral endothelial cells, pericytes, and astrocytes and leads to disruption of the blood-brain barrier (BBB) and ischemic cell damage (16-19). The evolution of downstream microvascular thrombosis is heterogeneous and continues for hours and is highly associated with the progression of ischemic neuronal death from reversible to irreversible damage $(14,16-18)$. Thus, the primary goal for treatment of ischemic stroke is to rapidly recanalyze the occluded blood vessels, reestablish CBF in the ischemic cerebral microvascular bed, preserve vascular integrity, and minimize neuronal death $(18,20)$.

During stroke recovery, ischemic brain undergoes a series of remodeling events that lead to limited spontaneous functional recovery (21). Relatively quiescent cerebral endothelial cells in preexisting blood vessels are activated, leading to angiogenesis in experimental stroke models and human ischemic brain (22-25). In addition to cerebral endothelial cells, circulating endothelial progenitor cells are also involved in the generation of new blood vessels (26). Stroke-induced angiogenesis not only occurs in the periinfarct regions, but also in the ventricular/subventricular zone (V/SVZ) of the lateral ventricles, a neurogenic region (27). At early stages of recovery, angiogenic vessels are permeable, but new vessels become less leaky and functional when they mature $(23,28)$. Increased angiogenesis is highly associated with improved neurological outcomes (10).

Neural stem cells are present in adult rodent brain in the V/ SVZ and in the subgranular zone of the dentate gyrus (29). These neural stem cells generate new neurons throughout life (29). Stroke increases neurogenesis in experimental animals (30-33), and an increase in neural progenitor cells and neuroblasts has also been observed in human ischemic brain (34-38). Strokeinduced neurogenesis couples to angiogenesis $(31,39-43)$. In the 
V/SVZ niche, neural stem cell proliferation induced by stroke is anatomically associated with activated cerebral endothelial cells (27). Newly generated neuroblasts in the V/SVZ migrate to periinfarct regions along cerebral blood vessels $(31,39-43)$. Blockage of cerebral angiogenesis impairs neurogenesis in the ischemic brain (31, 39-43). Neuroblasts appear to have a functional role in brain repair processes because depletion of neuroblasts exacerbates repair processes and worsens neurological outcome during stroke recovery (44).

In addition to neural progenitor cells, neural stem cells continuously generate oligodendrocyte progenitor cells (OPCs) that differentiate into myelinating oligodendrocytes (45-47). Mature oligodendrocytes are terminally differentiated and are vulnerable to cerebral ischemia. During brain repair processes, new oligodendrocytes generated by OPCs form myelin sheaths around the regenerated axons in periinfarct gray and white matter (48-51). Recent studies indicate that cerebral endothelial cells actively and mutually interact with oligodendrocytes to facilitate angiogenesis and oligodendrogenesis during repair after stroke (52).

\section{Exosomes and miRNAs}

Experimental studies have provided new insight into cellular and molecular mechanisms that underlie the intertwined brain remodeling processes. Both intracellular and intercellular molecules mediate the intertwined brain remodeling events via autocrine and paracrine signaling effects. For example, downregulation of miRNA-15a (miR-15a) in cerebral vessels in a mouse model of focal cerebral ischemia promotes stroke-induced angiogenesis in the periinfarct region by increasing FGF2 and VEGF levels (53). VEGF released by angiogenic endothelial cells also interacts with its receptor VEGFR2 in neural progenitor cells to promote their proliferation and neuronal differentiation (54). Moreover, cerebral endothelial cells in white matter are involved in regeneration of myelinating oligodendrocytes through brain-derived neurotrophic factor (BDNF) and FGF2 in injured brain (52). Stroke-induced limited axonal sprouting and remyelination in the periinfarct region are also regulated by miRNAs. In vitro and in vivo studies showed that stroke-induced downregulation of miR-9 and miR200 b expression in white matter mediates remyelination (55). Chondroitin sulfate proteoglycans (CSPGs) produced by reactive astrocytes inhibit axonal regrowth (56). Overexpression of the miR-17-92 cluster or miR-27a in cultured cortical neurons activates neuronal intrinsic growth signals by suppressing phosphatase and tensin homolog (PTEN) and ras homolog family member A (RhoA) signals, thereby overcoming the CSPG inhibitory effect $(57,58)$. In vivo studies of spinal cord injury in adult animals have shown that suppression of the PTEN signaling pathway within neurons enhances axonal sprouting even in the presence of CSPGs $(57,59)$. Thus, the miRNA and mRNA networks play a pivotal role in mediating brain-repair processes (60).

Emerging data suggest that exosome-mediated intercellular communication contributes to brain remodeling by transferring cargo from source cells to target cells (ref. 12 and Figure 1). Exosomes can be isolated from biofluids such as CSF and from the supernatant of cells cultured in exosome-free medium by centrifugation and other methods (61). Exosomes are generally enriched with tetraspanin proteins (CD63, CD81), the regulator of endosomal trafficking Alix, and the chaperone protein HSP70, although the content of exosomes varies with cell origin and physiological and pathological conditions $(13,61)$. Tetraspanins, Alix, and HSP70 have been used as exosomal markers (13). Proteomic and RNA analyses have demonstrated that exosomes carry cargoes of lipids, proteins, and RNAs, including mRNAs and miRNAs $(13,61)$; however, it is unclear how biological materials are loaded into individual exosomes.

All brain cells release exosomes (61). In vivo studies show that rodent and human brains release exosomes $(62,63)$. Compared with exosomes isolated from wild-type mouse brain, exosomes from brains of transgenic mice overexpressing human amyloid- $\beta$ $(\mathrm{A} \beta)$ precursor protein $(\mathrm{APP})$ contain high levels of full-length APP and C-terminal fragments of APP (62). Full-length and C-terminal fragments of APP were also detected within exosomes from brain tissue of an Alzheimer's disease (AD) patient (62). Fulllength APP cleavage by $\beta$-secretase occurs within endosomes, a fraction of $A \beta$ peptides is sorted to multivesicular bodies, and these $\mathrm{A} \beta$ peptides are released in association with exosomes (64). In addition, exosomes isolated from prefrontal cortices of patients with schizophrenia and bipolar disorder showed different profiles of exosomal miRNAs compared with exosomes from brains of control subjects (63). Collectively, these studies suggest that exosomes released by both human and mouse brains under disease conditions change profiles of exosomal cargo proteins and miRNAs and that exosomes enriched with neurotoxic C-terminal fragments of APP may contribute to the spread of A $\beta$ peptides to the brain. Although these in vivo studies are not able to indentify cellular sources of these exosomes, data from cultured cells suggest that exosomes released by neurons and astrocytes contain $A \beta$ peptides (see details below) $(64,65)$. It will be interesting to examine changes of brain exosomal cargo profiles and to investigate the role of their cargo in brain repair processes after stroke.

\section{Exosomes and cerebral angiogenesis}

In vitro and in vivo experiments have shown that exosomes from circulating endothelial progenitor cells transfer cargo mRNAs associated with the PI3K/Akt signaling pathway and proangiogenic miRNAs, such as miR-126 and miR-296, into recipient endothelial cells $(66,67)$. Within the recipient endothelial cells, these miRNAs activate the PI3K/Akt signaling pathway, leading to angiogenesis $(66,67)$. In the brain, exosomes from cultured glioblastoma cells induce angiogenesis by delivering their contents of proangiogenic proteins, mRNAs, and miRNAs into cerebral endothelial cells (68). Additionally, immortalized human brain microvascular endothelial cells secrete exosomes (69). Proteomic analysis has demonstrated that exosomes released by human cerebral endothelial cells contain 1,179 proteins, including several receptors that carry macromolecules across the BBB, such as transferrin receptor and insulin receptor (69). The role of these exosomal proteins has not been investigated, but interactions between cerebral endothelial exosomes and pericytes have been studied (70). Exosomes secreted by immortalized mouse cerebral endothelial cells stimulated by LPS and cytokines transferred cargo miRNAs and increased VEGF-B mRNA and protein levels in recipient cerebral vascular pericytes (70). VEGF-B and its receptor VEGFR-1 mediate angiogenesis (71). 


\section{Blood vessel}

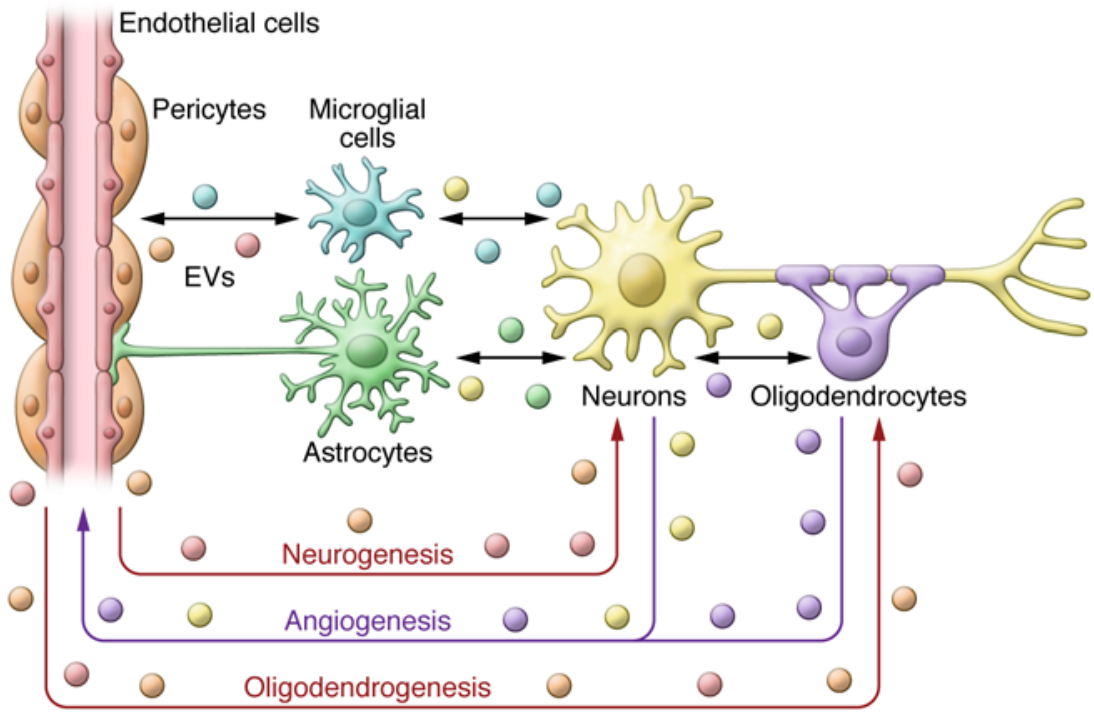

Figure 1. Potential of exosome-mediated intercellular communication in brain remodeling after stroke. Exosomes transfer proteins and RNAs to influence neurogenesis, angiogenesis, and oligodendrogenesis. EVs, extracellular vesicles.

\section{Exosomes and neurogenesis}

Neural stem cells in the V/SVZ of the adult rodent brain exist in a unique niche where they contact blood vessels, neighboring cells, and CSF, constantly exchanging molecular signals (79). We are just beginning to understand how intercellular molecular signals interact among the individual components within the niche. There is evidence that exosomes in CSF and neural stem cells mediate neural stem cell function and immune system function, respectively, by regulating intercellular pathways $(80,81)$. Exosomes isolated from embryonic CSF of rats and humans contain protein and miRNA components of the IGF signaling pathway (80). CSF-exosome cargoes, including both proteins and miRNAs, are highly conserved between rodent and human (80). Incubation of embryonic neural stem cells with CSF exosomes activated the IGF/mTORC1 pathway in the neural stem cells and promoted stem cell proliferation (80). Exposure of neural stem cells derived from V/SVZ neural stem cells of adult mouse

In addition, activation of the Notch signaling pathway between cerebral endothelial cells and pericytes is required for cerebral angiogenesis and BBB integrity (72). For example, Delta-like 4 (Dll4), a membrane-bound Notch ligand expressed by cerebral endothelial cells, stimulates Notch3 receptors on pericytes to keep the cerebral vascular structure quiescent (73). Exosomes released from human microvascular endothelial cells (HMECs) and human umbilical vein endothelial cells (HVECs) contain Dll4 proteins and have been shown to regulate development of angiogenesis $(74,75)$. The Notch signaling pathway interacts with the VEGF signaling pathway $(76,77)$. Together, these data suggest that cerebral endothelial exosomes could communicate with pericytes to mediate angiogenesis and to maintain $\mathrm{BBB}$ integrity through the VEGF and Notch signaling pathways.

Using a centrifugation approach, we have isolated exosomes released by cultured primary cerebral endothelial cells and neural progenitor cells harvested from nonischemic and ischemic animals. Proteomic and miRNA array analyses revealed that stroke substantially changed exosomal cargo proteins and miRNAs compared with exosomes from those nonischemic cells (78), indicating that stroke alters exosomal contents from cerebral endothelial cells and neural progenitor cells. Exosomes derived from ischemic neural progenitor cells promoted primary endothelial cell migration and capillary tube formation, whereas exosomes from ischemic cerebral endothelial cells enhanced neural progenitor cell proliferation and neuronal differentiation (78). These data suggest that exosomes secreted by cerebral endothelial cells and neural progenitor cells contribute to the observed coupling of neurogenesis and angiogenesis during brain repair processes after stroke. In addition, cerebral endothelial exosomes could also actively engage in brain remodeling by communicating with brain cells, including neurons and glia, and with remote cells in other organs during stroke recovery. to proinflammatory cytokines led to release of exosomes enriched with mRNAs encoding components of the IFN- $\gamma$ signaling pathway (81). These exosomes activated STAT1 signaling in recipient cells through exosome-associated IFN- $\gamma$ and its receptor IFNGR1 (81). Stroke activates innate and adaptive immune responses (82); thus, exosomes released by neural stem cells may also communicate with the immune system after stroke.

\section{Exosomes and neuronal plasticity}

Neurons and glia actively communicate with each other to coordinate axonal growth and myelination, and emerging data suggest that exosomes released by neurons and glia contribute to these processes $(61,83,84)$. Exosomes released by cultured cortical neurons carried the neuronal-specific protein L1 cell adhesion molecule (L1CAM) and the GluR2/3 subunits, but not the NR1 subunits, of glutamate receptors $(85,86)$. Increasing cytosolic calcium in neurons and neuronal depolarization augmented the secretion of exosomes (85-87). Notably, exosomes released by neurons contain alpha-amino-3-hydroxy-5-methyl-4-isoxazolepropionic acid (AMPA) receptors, while exosomes secreted from neurites of depolarized neurons are enriched with microtubuleassociated protein $1 \mathrm{~b}$ (MAP1b) and miRNAs that target genes involved in neurite plasticity $(86,87)$. Exosomes from neurons treated with a retinoic acid receptor $\beta 2(\operatorname{RAR} \beta)$ agonist had a dual effect on neurons and astrocytes to inactivate PTEN signaling, leading to enhancement of neurite outgrowth (88). RAR $\beta$ agonist inactivated cortical neuron PTEN signaling by releasing exosomes enriched with PTEN. These PTEN-enriched neuronal exosomes transferred PTEN proteins into astrocytes to suppress astrocyte proliferation (88). In addition to neurons, exosomes released by cortical neurons transferred miR-124 to astrocytes and increased the expression of the excitatory amino acid transporter GLT-1 in astrocytes, suggesting a role for neuronal exosomes in regulat- 
ing astrocyte function (89). AMPA receptors and MAP1b are key regulators of synaptic and dendritic plasticity and axonal spouting $(86,87,90)$. The astrocyte glutamate transporter GLT-1 in rodent regulates extracellular glutamate levels and modulates synaptic activation (89). Activation of AMPA receptors contributes to motor function recovery after stroke (91). Suppression of neuronal PTEN signals and reduction of an astrocyte scar promote axonal sprouting in adult CNS after spinal cord injury and stroke (57, 59, 92). Together, these data suggest that neuronal exosomes mediate synaptic and axonal plasticity by synaptic transfer of their cargo between neurons and by communication with astrocytes $(86,93)$, which potentially mediate axonal and synaptic remodeling in the ischemic brain.

\section{Exosomes and glia}

Exosomes derived from embryonic chicken astrocytes contain HSP70 (94). Treatment of astrocytes from newborn mice with high $\mathrm{KCl}$ concentrations led to release of exosomes enriched with synapsin, a synaptic vesicle-associated protein that enhances neurite growth and neuronal survival (95). A range of $\mathrm{KCl}$ concentrations can induce astrocyte secretion of exosomes in the ischemic brain $(95,96)$. Thus, such processes may occur in activated astrocytes after stroke.

Cultured oligodendrocytes secrete exosomes, and cytosolic calcium levels in oligodendrocytes regulate exosomal secretion (97). Proteomic analysis showed that oligodendrocyte-derived exosomes contained major myelin proteins, proteolipid protein (PLP), 2'3'-cyclic-nucleotide-phosphodiesterase (CNP), and myelin basic protein (MBP) (97). Glutamate from activated neurons also stimulates oligodendrocytes to release exosomes that carry MBP, PLP and sirtuin-2 (SIRT2) (98). These proteins inhibit myelination by activating Rho/ROCK/myosin signaling in recipient target cells (99). Transmission electron microscopic analysis showed the presence of $\mathrm{PLP}^{+}$multivesicular bodies in the periaxonal space (61), indicating that exosomes contribute to neuronalmediated coordination of myelination (61). In addition to myelination, exosomes from oligodendrocytes can be internalized by neurons and thereby improve neuronal viability under conditions of cell stress (98). In a model of in vitro ischemia, treatment of cortical neurons subjected to oxygen/glucose deprivation with oligodendrocyte-derived exosomes reduced ischemic neuronal death, which resulted from activation of signaling pathways in neurons by exosome cargo of superoxide dismutase, catalase, and other antioxidant enzymes (100). Thus, it appears that oligodendrocyte exosomes have multifaceted effects on neurons.

Microglia are a heterogeneous population of cells in the CNS and play a primary role in immune responses. Cultured primary microglia and microglia from a cell line released exosomes that contained the aminopeptidase CD13 and the lactate transporter monocarboxylate transporter 1 (MCT-1) (101). Microglial exosomes transferred CD13 to neurons, evoking neuropeptide degradation (101). Microglia can also take up exosomes secreted by other brain cells. In vitro and in vivo studies have demonstrated that exosomes from OPCs were preferentially internalized by microglia via a macropinocytotic mechanism (102). Compared with naive microglia, microglia challenged by IFN- $\gamma$ substantially reduced uptake of OPC exosomes (102). Microglia are also involved in the modulation of synaptic pruning, neurogenesis, and oligodendrogenesis (103-105). For example, SVZresident microglia mediate neuroblast migration, whereas in the hippocampus, IL-4- and IFN- $\gamma$-activated microglia promote oligodendrogenesis and neurogenesis, respectively $(104,105)$. These data imply that microglial exosomes regulate oligodendrogenesis and neurogenesis under physiological and pathophysiological conditions.

\section{Exosomes and cognitive deficits}

Stroke accelerates development of cognitive decline and $\mathrm{AD}$, which are characterized by the presence of $A \beta$ in the brain (18). $A \beta$ is secreted into the extracellular milieu, and an imbalance between production and clearance leads to accumulation of $A \beta$ (106). Exosomes secreted by cultured N2a neuroblastoma cells contained A $\beta$ (64). Internalization of neuronal exosomes by microglia enhanced microglial capability to take up and degrade $A \beta$ (107). In addition, statins, including simvastatin, stimulated microglia to secrete exosomes that were enriched with insulin-degrading enzyme (IDE), which is known to degrade $A \beta$ (108). These IDE-enriched exosomes promoted clearance of extracellular A $\beta$ peptides (108). These studies suggest that microglia clear $A \beta$ peptides by either internalization of exosomes derived from other cells or secretion of their own exosomes after statin treatment. Treatment of stroke and traumatic brain injury (TBI) with simvastatin enhanced ischemic brain repair processes, including axonal sprouting, and improved spontaneous object recognition and temporal order memory $(109,110)$. However, whether the statin treatment alters the ability of microglial exosomes to degrade $A \beta$ peptides in the injured brain remains to be investigated.

Exosomes derived from pathological cells are involved in the progression of neurological disorders. Exosomes derived from astrocytes of mice overexpressing mutant copper-zinc SOD1, a model of familial ALS, or astrocytes treated with A $\beta$ peptides have been shown to induce motor neuron death and astrocyte apoptosis, respectively, by shuttling mutant SOD1 and proapoptotic proteins into the recipient cells $(111,112)$.

\section{MSC-derived exosomes and therapies}

Cell therapies including bone mesenchymal stem cells (MSCs) improve neurological outcome after stroke and TBI $(10,18)$, and MSC therapy is in clinical trials for stroke (Table 1). Preclinical studies have demonstrated that MSCs promote angiogenesis, neurogenesis, and white matter remodeling in the injured brain by secreting factors to trigger the signaling pathways that are involved in brain repair. These findings indicate that paracrine effects of administered MSCs on brain parenchymal cells, but not cell replacement, underlie the benefits of MSC therapy $(10,18)$.

Compared with other cell types, cultured MSCs secrete a large quantity of exosomes (113). Emerging data from independent laboratories indicate that exosomes released from MSCs provide therapeutic benefits in stroke and TBI by modulating the brain microenvironment (114-119). Intravenous administration of MSC-derived exosomes to rats subjected to focal cerebral ischemia or TBI substantially improved neurological function by promoting neurovascular remodeling during stroke and TBI recovery $(116,117,119)$. Subsequently, the therapeutic 
Table 1. Clinical trials of MSC therapy for stroke

\begin{tabular}{|c|c|c|c|c|c|}
\hline Trial & Trial number & Purpose & Intervention arm & Phase & Primary end point \\
\hline $\begin{array}{l}\text { The STem Cell Application Researches and Trials In } \\
\text { NeuroloGy-2 (STARTING-2) Study }\end{array}$ & NCT01716481 & $\begin{array}{l}\text { Effect of MSCs on } \\
\text { neurologic deficits }\end{array}$ & IV autologous MSCS & 3 & Shift in $\mathrm{mRS}$ at $90 \mathrm{~d}$ \\
\hline $\begin{array}{l}\text { Autologous Bone Marrow Mesenchymal Stem Cell } \\
\text { Transplantation for Chronic Stroke }\end{array}$ & NCT01714167 & $\begin{array}{l}\text { Effect of MSCs on } \\
\text { chronic stroke }\end{array}$ & $\begin{array}{l}\text { Intracerebral injection } \\
\text { of autologous MSCs }\end{array}$ & 1 & $\begin{array}{l}\text { Change from baseline } \\
\text { in NIHSS at } 12 \text { mo }\end{array}$ \\
\hline $\begin{array}{l}\text { Autologous Bone Marrow Mesenchymal Stem Cell } \\
\text { Transplantation for Chronic Ischemic Stroke }\end{array}$ & NCT02564328 & $\begin{array}{l}\text { Effect of MSCs on } \\
\text { chronic stroke }\end{array}$ & IV autologous MSCs & 1 & $\begin{array}{l}\text { Change from baseline in } \\
\text { Fugl-Meyer scale at } 12 \mathrm{mo}\end{array}$ \\
\hline $\begin{array}{l}\text { Intravenous Autologous Mesenchymal Stem Cell } \\
\text { Transplantation to Treat Middle Cerebral Artery Infarct }\end{array}$ & NCT01461720 & $\begin{array}{l}\text { Effect of MSCs on } \\
\text { infarction }\end{array}$ & IV autologous MSCs & 2 & $\begin{array}{l}\text { Changes in NIHSS, mRS, } \\
\text { and BI at } 1 \mathrm{yr} \\
\text { Change infarct size at } 1 \mathrm{yr}\end{array}$ \\
\hline $\begin{array}{l}\text { Autologous Bone Marrow Stromal Cell } \\
\text { and Endothelial Progenitor Cell Transplantation } \\
\text { in Ischemic Stroke (AMETIS) }\end{array}$ & NCT01468064 & Safety of MSC therapy & $\begin{array}{l}\text { IV autologous MSCs, } \\
\text { IV autologous EPCs }\end{array}$ & 1 & $\begin{array}{l}\text { adverse events at } 1 \mathrm{yr} \text {, changes } \\
\text { in } \mathrm{mRS} \text { and the } \mathrm{Bl} \text { at } 1 \mathrm{yr}\end{array}$ \\
\hline
\end{tabular}

mRS, modified Rankin scale; NIHSS, NIH stroke scale; BI, Barthel index; EPCs, endothelial progenitor cells.

effect of MSC-derived exosomes has also been demonstrated by independent laboratories in the mouse subjected to stroke and TBI $(114,115)$. Systemic administration of MSC-derived extracellular vesicles to ischemic mice markedly reduced motor coordination deficits and enhanced angiogenesis and neurogenesis, while treatment of TBI mice with human MSCderived extracellular vesicles substantially preserved spatial leaning ability $(114,115)$. Improved neurological outcomes from these MSC-derived exosome studies are comparable to the therapeutic effect observed with MSC therapy, suggesting that MSC-derived exosome-mediated cell-cell communication may contribute to the therapeutic effect of the MSC therapy.

Exosomes transfer their cargo miRNAs to recipient cells (120, 121) and MSC-derived exosomes contain more than 700 miRNAs that are bound to argonaute2 (AGO2), a component of the RNAinduced silencing complex (RISC) $(122,123)$. The effect of engineered MSC-derived exosomes that carry elevated miRNAs on brain remodeling after stroke has been investigated in vitro and in vivo $(118,119)$. Treatment of stroke models with MSCs abolished stroke-induced downregulation of miR-133b in the ischemic brain (118). When MSCs were cultured with extracts harvested from ischemic brain tissues, they released exosomes enriched with miR-133b. Tailored MSC-derived exosomes with elevated or reduced miR-133b were harvested from the supernatant of MSCs transfected with lentiviral vectors carrying pre-miR-133b or antimiR-133b, respectively $(118,119)$. Intravenous administration of tailored MSC-derived exosomes with increased or decreased miR-133b to rats with stroke led to enhancement or exacerbation, respectively, of axonal remodeling and neurological function compared with naturally occurring MSC-derived exosomes (119). Connective tissue growth factor (CTGF) and RhoA are putative targets of miR-133b and are known to suppress neurite growth (118). In vitro, incubation of cortical neurons with miR-133belevated exosomes downregulated RhoA and enhanced neurite outgrowth, whereas treatment of astrocytes with miR-133b-elevated exosomes suppressed CTGF, which is mainly expressed by astrocytes $(119,124)$. Collectively, these data indicate that MSCderived exosomes may be used as vehicles to transport miRNAs that modulate genes in the recipient neurons and astrocytes.
Stroke induces immunosuppression in the peripheral blood, which exacerbates stroke outcome (125-127). In addition to interactions with brain cells, MSC-derived exosomes administered to ischemic mice appear to communicate with natural killer cells and lymphocytes in the peripheral blood to attenuate postischemic immunosuppression (114).

\section{HSC-exosomes and therapies}

Studies from myocardial ischemia have shown that engineered exosomes with elevated sonic hedgehog (Shh) derived from $\mathrm{CD}^{+} 4^{+}$hematopoietic stem cells (HSCs) transferred functional Shh and activated the Shh signaling pathway in recipient cells, enhancing angiogenesis in the border zone of infarction and preserving cardiac function (128). Shh (129-131) plays an important role in the regulation of adult neurogenesis under physiological and pathological conditions (132-135). These experiments suggest that exosomes can deliver functional proteins to modulate cellular function of recipient cells and that treatment of stroke with tailored Shh-exosomes could facilitate brain remodeling.

Exosomes can cross the BBB $(13,136)$. For example, intranasal administration of Odyssey 800 dye-labeled exosomes derived from a glioblastoma cell line led to distribution of fluorescent particles throughout the brain, mainly in the olfactory bulb in mice (136). Using the Cre-loxP system, studies have demonstrated that intrahippocampal injection of Cre-recombinase mRNA containing exosomes into mice with a ROSA26-lacZ reporter activated a lacZ reporter in the hippocampal neurons (98), indicating that Cre-recombinase mRNA within the exosomes activates the reporter gene in recipient neurons. Furthermore, intravenous injection of exosomes expressing a fusion protein consisting of the neuronspecific rabies virus glycoprotein (RVG) peptide with the exosomal membrane protein LAMP2B demonstrated targeting of neurons, microglia, and oligodendrocytes in the brain (137). Intravenous administration of the RVG peptide-expressing exosomes carrying siRNA against opioid receptor mu (MOR) enhanced the movement of exosomes across the BBB and inhibited MOR expression in the brain (138). These data suggest that exosomes not only cross the $\mathrm{BBB}$, but also deliver functional cargo to trigger gene expression in specific recipient cell types in the brain. 


\section{Perspectives}

Exosomes are emerging as important intercellular players in mediating neurorestorative events after stroke and neural injury (139-141). Preliminary work demonstrates that either naturally occurring or engineered exosomes derived from stem/progenitor cells provide therapeutic benefits (139-141). However, there are multiple unanswered questions and challenges in the development of exosome therapy including the following: (a) identification of the cellular signals by which the ischemic brain is able to affect the content and quantity of exosomes released by brain parenchymal cells and by remote organs, (b) understanding how exosomal cargo affects expression of endogenous genes and proteins in recipient cells of injured brain, (c) delineation of the specific cell types that are targeted by brain parenchymal cell-derived exosomes, and (d) knowledge of the effects of sex, age, and comorbidity on the cellular generation of exosomes and their cargo and the effect of sex, age and comorbid- ity in response to exosome treatment after stroke. Ongoing studies to investigate exosomes as a means of intercellular communication in ischemic brain will provide novel insights into the role of exosomes in the development of stroke pathogenesis and aid in the development of exosomal therapies to enhance stroke recovery.

\section{Acknowledgments}

The authors are supported by the following NIH grants: R01 NS 088656 (to M. Chopp), RO1 NS075156 (to Z.G. Zhang), and R01 NS079612 (to Z.G. Zhang). The content is solely the responsibility of the authors and does not necessarily represent the official views of the NIH.

Address correspondence to: Zheng Gang Zhang, Department of Neurology, 2799 West Grand Blvd., Detroit, Michigan 48202, USA. Phone: 313.916.5456; E-mail: zhazh@neuro.hfh.edu.
1. Lackland DT, et al. Factors influencing the decline in stroke mortality: a statement from the American Heart Association/American Stroke Association. Stroke. 2014;45(1):315-353.

2. [No authors listed]. Tissue plasminogen activator for acute ischemic stroke. N Engl J Med. 1995;333(24):1581-1587.

3. Hacke W, et al. Thrombolysis with alteplase 3 to 4. N Engl JMed. 2008;359(13):1317-1329.

4. Adeoye O, Hornung R, Khatri P, Kleindorfer D. Recombinant tissue-type plasminogen activator use for ischemic stroke in the United States: a doubling of treatment rates over the course of 5 years. Stroke. 2011;42(7):1952-1955.

5. Zivin JA. Acute stroke therapy with tissue plasminogen activator (tPA) since it was approved by the U. Ann Neurol. 2009;66(1):6-10.

6. Goyal M, et al. Randomized assessment of rapid endovascular treatment of ischemic stroke. NEngl JMed. 2015;372(11):1019-1030.

7. Campbell BC, et al. Endovascular therapy for ischemic stroke with perfusion-imaging selection. N Engl J Med. 2015;372(11):1009-1018.

8. Berkhemer OA, et al. A randomized trial of intraarterial treatment for acute ischemic stroke. N Engl J Med. 2015;372(1):11-20.

9. Lo EH, Dalkara T, Moskowitz MA. Mechanisms, challenges and opportunities in stroke. Nat Rev Neurosci. 2003;4(5):399-415.

10. Zhang ZG, Chopp M. Neurorestorative therapies for stroke: underlying mechanisms and translation to the clinic. Lancet Neurol. 2009;8(5):491-500.

11. Li Y, Liu Z, Xin H, Chopp M. The role of astrocytes in mediating exogenous cell-based restorative therapy for stroke. Glia. 2014;62(1):1-16.

12. Lai CP, Breakefield XO. Role of exosomes/ microvesicles in the nervous system and use in emerging therapies. Front Physiol. 2012;3:228.

13. Gyorgy B, Hung ME, Breakefield XO, Leonard JN. Therapeutic applications of extracellular vesicles: clinical promise and open questions. Annu Rev Pharmacol Toxicol. 2015;55:439-464.

14. Zhang ZG, et al. Dynamic platelet accumulation at the site of the occluded middle cerebral artery and in downstream microvessels is associated with loss of microvascular integrity after embolic middle cerebral artery occlusion. Brain Res. 2001;912(2):181-194.

15. Furie B, Furie BC. Mechanisms of thrombus formation. N Engl J Med. 2008;359(9):938-949.

16. Garcia JH, et al. Progression from ischemic injury to infarct following middle cerebral artery occlusion in the rat. Am J Pathol.1993;142(2):623-635.

17. Zhang ZG, et al. Cerebral microvascular obstruction by fibrin is associated with upregulation of PAI-1 acutely after onset of focal embolic ischemia in rats. J Neurosci. 1999;19(24):10898-10907.

18. Moskowitz MA, Lo EH, Iadecola C. The science of stroke: mechanisms in search of treatments. Neuron. 2010;67(2):181-198.

19. Rosenberg GA. Neurological diseases in relation to the blood-brain barrier. J Cereb Blood Flow Metab. 2012;32(7):1139-1151.

20. Ginsberg MD. Current status of neuroprotection for cerebral ischemia: synoptic overview. Stroke. 2009;40(3 suppl):S111-S114.

21. Nudo RJ. Postinfarct cortical plasticity and behavioral recovery. Stroke. 2007;38(2 suppl):840-845.

22. Krupinski J, Kaluza J, Kumar P, Kumar S, Wang JM. Role of angiogenesis in patients with cerebral ischemic stroke. Stroke. 1994;25(9):1794-1798.

23. Zhang ZG, et al. VEGF enhances angiogenesis and promotes blood-brain barrier leakage in the ischemic brain. J Clin Invest. 2000;106(7):829-838.

24. Risau W. Mechanisms of angiogenesis. Nature. 1997;386(6626):671-674.

25. Carmeliet P. VEGF gene therapy: stimulating angiogenesis or angioma-genesis? Nat Med. 2000;6(10):1102-1103.

26. Zhang ZG, Zhang L, Jiang Q, Chopp M. Bone marrow-derived endothelial progenitor cells participate in cerebral neovascularization after focal cerebral ischemia in the adult mouse. Circ Res. 2002;90(3):284-288.

27. Zhang RL, et al. Stroke increases neural stem cells and angiogenesis in the neurogenic niche of the adult mouse. PLoS One. 2014;9(12):e113972.

28. Plate KH. Mechanisms of angiogenesis in the brain. J Neuropathol Exp Neurol. 1999;58(4):313-320.

29. Miller FD, Gauthier-Fisher A. Home at last: neural stem cell niches defined. Cell Stem Cell. 2009;4(6):507-510.

30. Iwai M, et al. Temporal profile of stem cell division, migration, and differentiation from subventricular zone to olfactory bulb after transient forebrain ischemia in gerbils. J Cereb Blood Flow Metab. 2003;23(3):331-341.

31. Ohab JJ, Fleming S, Blesch A, Carmichael ST. A neurovascular niche for neurogenesis after stroke. J Neurosci. 2006;26(50):13007-13016.

32. Yamashita T, et al. Subventricular zone-derived neuroblasts migrate and differentiate into mature neurons in the post-stroke adult striatum. JNeurosci. 2006;26(24):6627-6636.

33. Felling RJ, et al. Neural stem/progenitor cells participate in the regenerative response to perinatal hypoxia/ischemia. J Neurosci. 2006;26(16):4359-4369.

34. Jin K, et al. Evidence for stroke-induced neurogenesis in the human brain. Proc Natl Acad Sci USA. 2006;103(35):13198-13202.

35. Macas J, Nern C, Plate KH, Momma S. Increased generation of neuronal progenitors after ischemic injury in the aged adult human forebrain. JNeurosci. 2006;26(50):13114-13119.

36. Minger SL, Ekonomou A, Carta EM, Chinoy A, Perry RH, Ballard CG. Endogenous neurogenesis in the human brain following cerebral infarction. Regen Med. 2007;2(1):69-74.

37. Curtis MA, Kam M, Faull RL. Neurogenesis in humans. Eur J Neurosci. 2011;33(6):1170-1174.

38. Marti-Fabregas J, et al. Proliferation in the human ipsilateral subventricular zone after ischemic stroke. Neurology. 2010;74(5):357-365.

39. Silva-Vargas V, Crouch EE, Doetsch F. Adult neural stem cells and their niche: a dynamic duo during homeostasis, regeneration, and aging. Curr Opin Neurobiol. 2013;23(6):935-942.

40. Tran PB, Ren D, Veldhouse TJ, Miller RJ. Chemokine receptors are expressed widely by embryonic and adult neural progenitor cells. J Neurosci Res. 2004;76(1):20-34.

41. Robin AM, et al. Stromal cell-derived factor $1 \alpha$ mediates neural progenitor cell motility after focal cerebral ischemia. J Cereb Blood Flow Metab. 2006;26(1):125-134.

42. Zhang RL, et al. Patterns and dynamics of subven- 
tricular zone neuroblast migration in the ischemic striatum of the adult mouse. JCereb Blood Flow Metab. 2009;29(7):1240-1250.

43. Zhang RL, et al. Neuroblast division during migration toward the ischemic striatum: a study of dynamic migratory and proliferative characteristics of neuroblasts from the subventricular zone. JNeurosci. 2007;27(12):3157-3162.

44. Wang X, Mao X, Xie L, Sun F, Greenberg DA, Jin K. Conditional depletion of neurogenesis inhibits long-term recovery after experimental stroke in mice. PLoS One. 2012;7(6):e38932.

45. Young KM, et al. Oligodendrocyte dynamics in the healthy adult CNS: evidence for myelin remodeling. Neuron. 2013;77(5):873-885.

46. Fields RD. White matter in learning, cognition and psychiatric disorders. Trends Neurosci. 2008;31(7):361-370.

47. Zatorre RJ, Fields RD, Johansen-Berg H. Plasticity in gray and white: neuroimaging changes in brain structure during learning. Nat Neurosci. 2012;15(4):528-536.

48. Zhang RL, et al. Sildenafil enhances neurogenesis and oligodendrogenesis in ischemic brain of middle-aged mouse. PLoS One. 2012;7(10):e48141.

49. Zhang RL, et al. Ascl1 lineage cells contribute to ischemia-induced neurogenesis and oligodendrogenesis. J Cereb Blood Flow Metab. 2011;31(2):614-625.

50. Ueno Y, et al. Axonal outgrowth and dendritic plasticity in the cortical peri-infarct area after experimental stroke. Stroke. 2012;43(8):2221-2228.

51. Gregersen R, Christensen T, Lehrmann E, Diemer $\mathrm{NH}$, Finsen B. Focal cerebral ischemia induces increased myelin basic protein and growthassociated protein- 43 gene transcription in peri-infarct areas in the rat brain. Exp Brain Res. 2001;138(3):384-392.

52. Miyamoto N, Pham LD, Seo JH, Kim KW, Lo EH, Arai K. Crosstalk between cerebral endothelium and oligodendrocyte. Cell Mol Life Sci. 2014;71(6):1055-1066.

53. Yin KJ, Hamblin M, Chen YE. Angiogenesisregulating microRNAs and Ischemic Stroke. Curr Vasc Pharmacol. 2015;13(3):352-365.

54 . Teng $\mathrm{H}$, et al. Coupling of angiogenesis and neurogenesis in cultured endothelial cells and neural progenitor cells after stroke. JCereb Blood Flow Metab. 2008;28(4):764-771.

55. Buller B, et al. Regulation of serum response factor by miRNA-200 and miRNA- 9 modulates oligodendrocyte progenitor cell differentiation. Glia. 2012;60(12):1906-1914.

56. Gherardini L, Gennaro M, Pizzorusso T. Perilesional treatment with chondroitinase $\mathrm{ABC}$ and motor training promote functional recovery after stroke in rats. Cereb Cortex. 2015;25(1):202-212.

57. Zhang Y, et al. MicroRNAs in the axon locally mediate the effects of chondroitin sulfate proteoglycans and cGMP on axonal growth. Dev Neurobiol. 2015;75(12):1402-1419.

58. Zhang Y, et al. The MicroRNA-17-92 cluster enhances axonal outgrowth in embryonic cortical neurons. J Neurosci. 2013;33(16):6885-6894.

59. Park KK, et al. Promoting axon regeneration in the adult CNS by modulation of the PTEN/mTOR pathway. Science. 2008;322(5903):963-966.

60. He X, Yu Y, Awatramani R, Lu QR. Unwrap- ping myelination by microRNAs. Neuroscientist. 2012;18(1):45-55.

61. Fruhbeis C, Frohlich D, Kuo WP, Kramer-Albers EM. Extracellular vesicles as mediators of neuron-glia communication. Front Cell Neurosci. 2013;7:182.

62. Perez-Gonzalez R, Gauthier SA, Kumar A, Levy E. The exosome secretory pathway transports amyloid precursor protein carboxyl-terminal fragments from the cell into the brain extracellular space. J Biol Chem. 2012;287(51):43108-43115.

63. Banigan MG, et al. Differential expression of exosomal microRNAs in prefrontal cortices of schizophrenia and bipolar disorder patients. PLoS One. 2013;8(1):e48814.

64. Rajendran L, et al. Alzheimer's disease $\beta$-amyloid peptides are released in association with exosomes. Proc Natl Acad Sci U S A. 2006;103(30):11172-11177.

65. Dinkins MB, Dasgupta S, Wang G, Zhu G, Bieberich $\mathrm{E}$. Exosome reduction in vivo is associated with lower amyloid plaque load in the 5XFAD mouse model of Alzheimer's disease. Neurobiol Aging. 2014;35(8):1792-1800.

66. Cantaluppi V, et al. Microvesicles derived from endothelial progenitor cells enhance neoangiogenesis of human pancreatic islets. Cell Transplant. 2012;21(6):1305-1320

67. Deregibus MC, et al. Endothelial progenitor cell derived microvesicles activate an angiogenic program in endothelial cells by a horizontal transfer of mRNA. Blood. 2007;110(7):2440-2448.

68. Skog J, et al. Glioblastoma microvesicles transport RNA and proteins that promote tumour growth and provide diagnostic biomarkers. Nat Cell Biol. 2008;10(12):1470-1476.

69. Haqqani AS, Delaney CE, Tremblay TL, Sodja C, Sandhu JK, Stanimirovic DB. Method for isolation and molecular characterization of extracellular microvesicles released from brain endothelial cells. Fluids Barriers CNS. 2013;10(1):4.

70. Yamamoto S, et al. Inflammation-induced endothelial cell-derived extracellular vesicles modulate the cellular status of pericytes. $S c i$ Rep. 2015;5:8505.

71. Takahashi H, Shibuya M. The vascular endothelial growth factor (VEGF)/VEGF receptor system and its role under physiological and pathological conditions. Clin Sci (Lond). 2005;109(3):227-241.

72. Winkler EA, Bell RD, Zlokovic BV. Central nervous system pericytes in health and disease. Nat Neurosci. 2011;14(11):1398-1405.

73. Schulz GB, et al. Cerebral cavernous malformation-1 protein controls DLL4-Notch3 signaling between the endothelium and pericytes. Stroke. 2015;46(5):1337-1343.

74. Sheldon H, et al. New mechanism for Notch signaling to endothelium at a distance by $\Delta$-like 4 incorporation into exosomes. Blood. 2010;116(13):2385-2394.

75. Sharghi-Namini S, Tan E, Ong LL, Ge R, Asada HH Dll4-containing exosomes induce capillary sprout retraction in a 3D microenvironment. Sci Rep. 2014;4:4031.

76. Tammela T, et al. Blocking VEGFR-3 suppresses angiogenic sprouting and vascular network formation. Nature. 2008;454(7204):656-660.

77. Taylor KL, Henderson AM, Hughes CC. Notch activation during endothelial cell network formation in vitro targets the basic HLH transcription factor HESR-1 and downregulates VEGFR-2/KDR expression. Microvasc Res. 2002;64(3):372-383.

78. Pan W, et al. Exosomes derived from ischemic cerebral endothelial cells and neural progenitor cells enhance neurogenesis and angiogenesis. Stroke. 2016;47(suppl 1):AWMP39.

79. Ihrie RA, Alvarez-Buylla A. Lake-front property: a unique germinal niche by the lateral ventricles of the adult brain. Neuron. 2011;70(4):674-686.

80. Feliciano DM, Zhang S, Nasrallah CM, Lisgo SN, Bordey A. Embryonic cerebrospinal fluid nanovesicles carry evolutionarily conserved molecules and promote neural stem cell amplification. PLoS One. 2014;9(2):e88810.

81. Cossetti C, et al. Extracellular vesicles from neural stem cells transfer IFN-gamma via Ifngr1 to activate Stat1 signaling in target cells. Mol Cell. 2014;56(2):193-204.

82. Famakin BM. The immune response to acute focal cerebral ischemia and associated poststroke immunodepression: a focused review. Aging Dis. 2014;5(5):307-326.

83. Higa GS, de Sousa E, Walter LT, Kinjo ER, Resende RR, Kihara AH. MicroRNAs in neuronal communication. Mol Neurobiol. 2014;49(3):1309-1326.

84. Kawikova I, Askenase PW. Diagnostic and therapeutic potentials of exosomes in CNS diseases. Brain Res. 2015;1617:63-71.

85. Faure J, et al. Exosomes are released by cultured cortical neurones. Mol Cell Neurosci. 2006;31(4):642-648

86. Lachenal G, et al. Release of exosomes from differentiated neurons and its regulation by synaptic glutamatergic activity. Mol Cell Neurosci. 2011;46(2):409-418.

87. Goldie BJ, et al. Activity-associated miRNA are packaged in Map1b-enriched exosomes released from depolarized neurons. Nucleic Acids Res. 2014;42(14):9195-9208.

88. Goncalves MB, et al. Neuronal RAR $\beta$ signaling modulates PTEN activity directly in neurons and via exosome transfer in astrocytes to prevent glial scar formation and induce spinal cord regeneration. J Neurosci. 2015;35(47):15731-15745.

89. Morel L, et al. Neuronal exosomal miRNAdependent translational regulation of astroglial glutamate transporter GLT1. J Biol Chem. 2013;288(10):7105-7116.

90. Dajas-Bailador F, Bonev B, Garcez P, Stanley P, Guillemot F, Papalopulu N. microRNA-9 regulates axon extension and branching by targeting Map1b in mouse cortical neurons [published online ahead of print April 8, 2012]. Nat Neurosci. doi:10.1038/nn.3082.

91. Clarkson AN, Overman JJ, Zhong S, Mueller R, Lynch G, Carmichael ST. AMPA receptor-induced local brain-derived neurotrophic factor signaling mediates motor recovery after stroke. J Neurosci. 2011;31(10):3766-3775.

92. Shen LH, Li Y, Gao Q, Savant-Bhonsale S, Chopp M. Down-regulation of neurocan expression in reactive astrocytes promotes axonal regeneration and facilitates the neurorestorative effects of bone marrow stromal cells in the ischemic rat brain. Glia. 2008;56(16):1747-1754. 
93. Edelstein L, Smythies J. The role of epigeneticrelated codes in neurocomputation: dynamic hardware in the brain. Philos Trans R Soc Lond B Biol Sci. 2014;369(1652):20130519.

94. Taylor AR, Robinson MB, Gifondorwa DJ, Tytell $\mathrm{M}$, Milligan CE. Regulation of heat shock protein 70 release in astrocytes: role of signaling kinases. Dev Neurobiol. 2007;67(13):1815-1829.

95. Wang S, et al. Synapsin I is an oligomannose-carrying glycoprotein, acts as an oligomannose-binding lectin, and promotes neurite outgrowth and neuronal survival when released via glia-derived exosomes. JNeurosci. 2011;31(20):7275-7290.

96. Lauritzen M, Dreier JP, Fabricius M, Hartings JA, Graf R, Strong AJ. Clinical relevance of cortical spreading depression in neurological disorders: migraine, malignant stroke, subarachnoid and intracranial hemorrhage, and traumatic brain injury. J Cereb Blood Flow Metab. 2011;31(1):17-35.

97. Kramer-Albers EM, et al. Oligodendrocytes secrete exosomes containing major myelin and stressprotective proteins: Trophic support for axons? Proteomics Clin Appl. 2007;1(11):1446-1461.

98. Fruhbeis C, et al. Neurotransmitter-triggered transfer of exosomes mediates oligodendrocyte-neuron communication. PLoS Biol. 2013;11(7):e1001604.

99. Bakhti M, Winter C, Simons M. Inhibition of myelin membrane sheath formation by oligodendrocyte-derived exosome-like vesicles. J Biol Chem. 2011;286(1):787-796.

100.Frohlich D, et al. Multifaceted effects of oligodendroglial exosomes on neurons: impact on neuronal firing rate, signal transduction and gene regulation. Philos Trans R Soc Lond B Biol Sci. 2014;369(1652):2013510.

101.Potolicchio I, et al. Proteomic analysis of microglia-derived exosomes: metabolic role of the aminopeptidase $\mathrm{CD} 13$ in neuropeptide catabolism. JImmunol. 2005;175(4):2237-2243.

102. Fitzner D, et al. Selective transfer of exosomes from oligodendrocytes to microglia by macropinocytosis. J Cell Sci. 2011;124(pt 3):447-458.

103. Sato K. Effects of Microglia on Neurogenesis. Glia. 2015;63(8):1394-1405.

104. Ribeiro Xavier AL, Kress BT, Goldman SA, Lacerda de Menezes JR, Nedergaard M. A distinct population of microglia supports adult neurogenesis in the subventricular zone. JNeurosci. 2015;35(34):11848-11861.

105. Butovsky O, et al. Microglia activated by IL-4 or IFN- $\gamma$ differentially induce neurogenesis and oligodendrogenesis from adult stem/progenitor cells. Mol Cell Neurosci. 2006;31(1):149-160.

106. Querfurth HW, LaFerla FM. Alzheimer's disease. N Engl J Med. 2010;362(4):329-344.

107. Yuyama K, Sun H, Mitsutake S, Igarashi Y. Sphingolipid-modulated exosome secretion promotes clearance of amyloid- $\beta$ by microglia. J Biol Chem. 2012;287(14):10977-10989.

108. Tamboli IY, et al. Statins promote the degradation of extracellular amyloid $\beta$-peptide by microglia via stimulation of exosome-associated insulindegrading enzyme (IDE) secretion. J Biol Chem. 2010;285(48):37405-37414.

109. Darwish H, Mahmood A, Schallert T, Chopp M, Therrien B. Simvastatin and environmental enrichment effect on recognition and temporal order memory after mild-to-moderate traumatic brain injury. Brain Inj. 2014;28(2):211-226.

110. Chen J, et al. Statins induce angiogenesis, neurogenesis, and synaptogenesis after stroke. Ann Neurol. 2003;53(6):743-751.

111. Basso M, et al. Mutant copper-zinc superoxide dismutase (SOD1) induces protein secretion pathway alterations and exosome release in astrocytes: implications for disease spreading and motor neuron pathology in amyotrophic lateral sclerosis. J Biol Chem. 2013;288(22):15699-15711.

112.Wang G, et al. Astrocytes secrete exosomes enriched with proapoptotic ceramide and prostate apoptosis response 4 (PAR-4): potential mechanism of apoptosis induction in Alzheimer disease (AD). J Biol Chem. 2012;287(25):21384-21395.

113. Yeo RW, et al. Mesenchymal stem cell: an efficient mass producer of exosomes for drug delivery. $A d v$ Drug Deliv Rev. 2013;65(3):336-341.

114. Doeppner TR, et al. Extracellular vesicles improve post-stroke neuroregeneration and prevent postischemic immunosuppression. Stem Cells Transl Med. 2015;4(10):1131-1143.

115. Kim DK, Nishida H, An SY, Shetty AK, Bartosh TJ, Prockop DJ. Chromatographically isolated $\mathrm{CD} 63^{+} \mathrm{CD} 81^{+}$extracellular vesicles from mesenchymal stromal cells rescue cognitive impairments after TBI. Proc Natl Acad Sci U S A. 2016;113(1):170-175.

116. Zhang Y, et al. Effect of exosomes derived from multipluripotent mesenchymal stromal cells on functional recovery and neurovascular plasticity in rats after traumatic brain injury. J Neurosurg. 2015;122(4):856-867.

117. Xin H, Li Y, Cui Y, Yang JJ, Zhang ZG, Chopp M. Systemic administration of exosomes released from mesenchymal stromal cells promote functional recovery and neurovascular plasticity after stroke in rats. J Cereb Blood Flow Metab. 2013;33(11):1711-1715.

118. Xin H, et al. Exosome-mediated transfer of miR-133b from multipotent mesenchymal stromal cells to neural cells contributes to neurite outgrowth. Stem Cells. 2012;30(7):1556-1564.

119. Xin H, et al. MiR-133b promotes neural plasticity and functional recovery after treatment of stroke with multipotent mesenchymal stromal cells in rats via transfer of exosome-enriched extracellular particles. Stem Cells. 2013;31(12):2737-2746.

120. Penfornis P, Vallabhaneni KC, Whitt J, Pochampally R. Extracellular vesicles as carriers of microRNA, proteins and lipids in tumor microenvironment. Int J Cancer. 2016;138(1):14-21.

121. Vallabhaneni KC, et al. Extracellular vesicles from bone marrow mesenchymal stem/stromal cells transport tumor regulatory microRNA, proteins, and metabolites. Oncotarget. 2015;6(7):4953-4967.

122.Zhang Y, Chopp M, Katakowski M, Liu X, Zhang Z. Exosomes derived from mesenchymal stromal cells promote axonal outgrowth. Stroke. 2015;46:A67.

123. Zhang ZG, Chopp M. Promoting brain remodeling to aid in stroke recovery. Trends Mol Med. 2015;21(9):543-548.
124.Jones EV, Bouvier DS. Astrocyte-secreted matricellular proteins in CNS remodelling during development and disease. Neural Plast. 2014;2014:321209.

125. Offner H, Vandenbark AA, Hurn PD. Effect of experimental stroke on peripheral immunity: CNS ischemia induces profound immunosuppression. Neuroscience. 2009;158(3):1098-1111.

126.Prass K, et al. Stroke-induced immunodeficiency promotes spontaneous bacterial infections and is mediated by sympathetic activation reversal by poststroke Thelper cell type 1-like immunostimulation. JExp Med. 2003;198(5):725-736.

127. Wong CH, Jenne CN, Lee WY, Leger C, Kubes P. Functional innervation of hepatic iNKT cells is immunosuppressive following stroke. Science. 2011;334(6052):101-105.

128. Mackie AR, et al. Sonic hedgehog-modified human $\mathrm{CD} 34^{+}$cells preserve cardiac function after acute myocardial infarction. Circ Res. 2012;111(3):312-321.

129. Ruiz i Altaba A, Sanchez P, Dahmane N. Gli and hedgehog in cancer: tumours, embryos and stem cells. Nat Rev Cancer. 2002;2(5):361-372.

130. Goetz JA, Suber LM, Zeng X, Robbins DJ. Sonic Hedgehog as a mediator of long-range signaling. Bioessays. 2002;24(2):157-165.

131. Roberts DJ, Johnson RL, Burke AC, Nelson CE, Morgan BA, Tabin C. Sonic hedgehog is an endodermal signal inducing Bmp-4 and Hox genes during induction and regionalization of the chick hindgut. Development. 1995;121(10):3163-3174.

132. Androutsellis-Theotokis A, et al. Notch signalling regulates stem cell numbers in vitro and in vivo. Nature. 2006;442(7104):823-826.

133. Palma V, et al. Sonic hedgehog controls stem cell behavior in the postnatal and adult brain. Development. 2005;132(2):335-344.

134. Liu XS, et al. MicroRNA-17-92 cluster mediates the proliferation and survival of neural progenitor cells after stroke. J Biol Chem. 2013;288(18):12478-12488.

135. Wang L, et al. The Sonic hedgehog pathway mediates carbamylated erythropoietinenhanced proliferation and differentiation of adult neural progenitor cells. J Biol Chem. 2007;282(44):32462-32470.

136.Zhuang X, et al. Treatment of brain inflammatory diseases by delivering exosome encapsulated anti-inflammatory drugs from the nasal region to the brain. Mol Ther. 2011;19(10):1769-1779.

137. Alvarez-Erviti L, Seow Y, Yin H, Betts C, Lakhal S, Wood MJ. Delivery of siRNA to the mouse brain by systemic injection of targeted exosomes. Nat Biotechnol. 2011;29(4):341-345.

138. Liu Y, et al. Targeted exosome-mediated delivery of opioid receptor Mu siRNA for the treatment of morphine relapse. Sci Rep. 2015;5:17543.

139. Xin H, Li Y, Chopp M. Exosomes/miRNAs as mediating cell-based therapy of stroke. Front Cell Neurosci. 2014;8:377.

140.Liu XS, Chopp M, Zhang RL, Zhang ZG. MicroRNAs in cerebral ischemia-induced neurogenesis. J Neuropathol Exp Neurol. 2013;72(8):718-722.

141. Zhang R, Chopp M, Zhang ZG. Oligodendrogenesis after cerebral ischemia. Front Cell Neurosci. 2013;7:201. 